\title{
Feasibility of acoustic monitoring of strength drop precursory to earthquake occurrence
}

\author{
Nobuki Kame ${ }^{1 *}$, Kohei Nagata1,2, Masao Nakatani ${ }^{1}$ and Tetsuya Kusakabe
}

\begin{abstract}
Rate- and state-dependent friction law (RSF), proposed on the basis of laboratory experiments, has been extensively applied to modeling of earthquake stick-slip cycles. A simple spring-slider model obeying RSF predicts a significant decrease of the frictional strength $\Phi$ (the state of contact) that is localized within a few years preceding the earthquake occurrence. On the other hand, recent laboratory experiments successfully monitored the history of the strength by simultaneously measuring the P-wave transmissivity $|T|$ across the frictional interface using a 1-MHz transducer. This suggests a possibility of earthquake forecast by monitoring the strength of a natural fault by acoustic methods. The present paper explores the feasibility of such monitoring in the field on the basis of the physics of RSF combined with the linear slip model (LSM) employed in the classical acoustic methodology for monitoring an imperfectly welded interface. The characteristic frequency $f_{c}$, around which $|T|$ (or reflectivity $|R|$ ) has a good sensitivity to the interface strength, is shown to be proportional to the strength and inversely proportional to the representative scale of real contacts. For natural faults, $f_{c}$ is estimated to be 1 to $100 \mathrm{~Hz}$, which is practicable in the field. The changes of $|T|$ and $|R|$ depend on the ratio of the strength drop to the absolute strength level, the latter of which is not constrained by RSF simulations. Expected changes in wave amplitude in the preslip period would be several percent for strong faults and several tens percent for weak faults, which may be detectable by acoustic methods such as seismic reflection surveys.
\end{abstract}

Keywords: Fault strength; Earthquake cycle; Rate- and state-dependent friction; Precursor; Linear slip model; Acoustic monitoring; Reflection survey

\section{Background}

Empirical friction laws called rate- and state-dependent friction (RSF) (e.g., Dieterich 1979; Ruina 1983) have been extensively applied to modeling of earthquake stick-slip cycles because RSF is based on laboratory rock friction tests and therefore considered to be a realistic friction law. A simple spring-slider earthquake cycle model obeying RSF has predicted a significant decrease of the frictional strength $\Phi$ prior to the occurrence of an earthquake (Kame et al. 2012a). Recent laboratory experiments successfully monitored the histories of the strength including as large a precursor as predicted by Kame et al.s simulations, by simultaneously measuring acoustic transmissivity $|T|$ across the frictional interface using a $1-\mathrm{MHz}$ transducer (Nagata et al. 2008, 2012) though their experiments were artificially imposed hold-slide cycles, not

\footnotetext{
*Correspondence: kame@eri.u-tokyo.ac.jp

${ }^{1}$ Earthquake Research Institute, the University of Tokyo, 1-1-1 Yayoi, Bunkyo-ku, Tokyo 113-0032, Japan

Full list of author information is available at the end of the article
}

spontaneous stick-slip cycles (Figure 1). Two fundamental questions need to be addressed to assess the feasibility of acoustic monitoring of natural faults. One is whether the sensitive frequency range for monitoring is within a range usable in the field; the experimental $1 \mathrm{MHz}$ is obviously out of range. The other is the magnitude of the change in wave amplitude resulting from the expected strength drop. For this purpose, we theoretically relate the strength of RSF faults with the linear slip model (LSM) widely employed in the acoustic methodology. By using the relationship, fairly optimistic answers have been obtained to both questions, in stark contrast to the generally pessimistic assessment of the detectability of the elastic deformation of the surrounding rock caused by preslip.

\section{The concept of strength in RSF}

'Strength' involved in RSF is a concept central to the present paper. We here extend the concept following Nakatani (2001). RSF consists of two equations bearing

\section{黑 Springer}

(c) 2014 Kame et al: licensee Springer. This is an Open Access article distributed under the terms of the Creative Commons Attribution License (http://creativecommons.org/licenses/by/2.0), which permits unrestricted use, distribution, and reproduction in any medium, provided the original work is properly credited. 


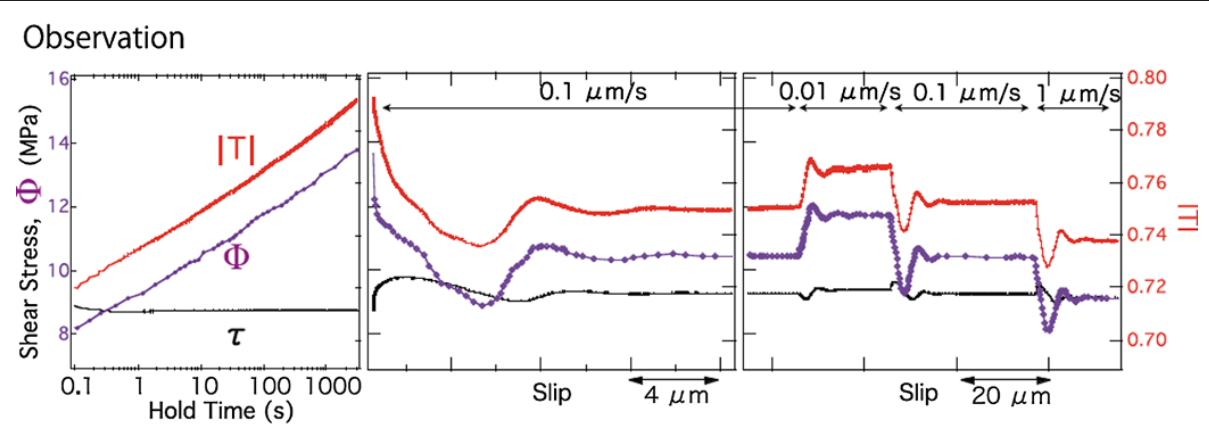

Simulation

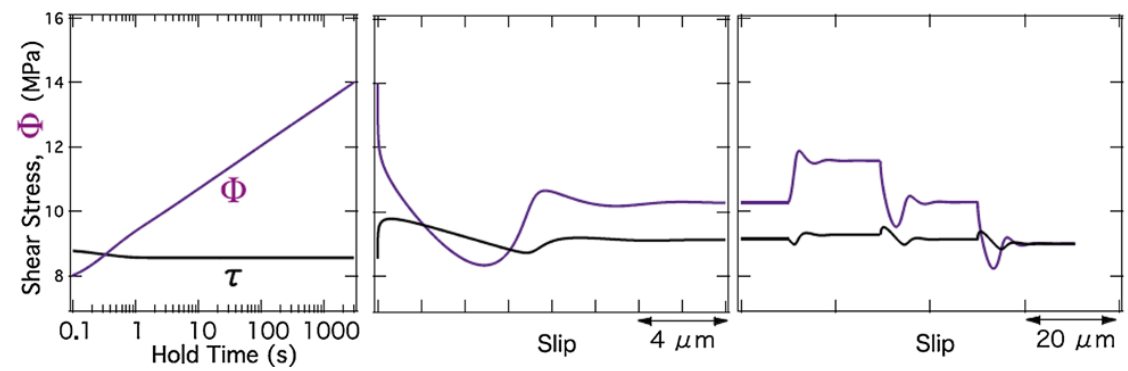

Figure 1 Comparison of $\boldsymbol{\Phi}$ (state, or strength) and $\boldsymbol{\tau}$ (shear stress). Between observations and simulations for various types of experiments, such as a hold under a constant shear stress (left column), reloading after the hold (middle column), steady-state sliding at different velocities, and transience between them (right column) from Figure Sixteen of Nagata et al. (2012). |T| is the acoustic transmissivity measured by the amplitude of P wave that has traveled across the interface. The simulations are based on the Nagata law.

logically separate roles (Nakatani 2001). The first equation is called the constitutive law, which describes the relationship between the applied shear stress $\tau$ and the slip rate $V$ under the given physical state of the interface specified by a state variable $\Phi$ as

$$
V=V_{*} \exp \left(\frac{\tau-\Phi}{a \sigma}\right), \quad \text { or } \quad \tau=\Phi+a \sigma \ln \left(\frac{V}{V_{*}}\right)
$$

where $V_{*}$ is an arbitrarily chosen reference velocity, $\sigma$ is the normal stress, and $a$ is a nondimensional positive parameter called the direct effect coefficient. $\Phi$ is often associated with the real contact area $S$, which can be monitored independently by, for example, optical or acoustic methods (Dieterich and Kilgore 1994; 1996; Nagata et al. 2008; 2012). The exponential form of the constitutive law implies that the physical mechanism behind it is a thermally activated creep driven by very high shear stresses at the real contacts of an imperfectly welded interface (Heslot et al. 1994; Nakatani 2001). Macroscopically, $\Phi$ in Equation 1 can be regarded as an extension of classical frictional strength because $\Phi$ is the shear stress required to move the interface at a reference velocity $V_{*}$, noticing that a larger applied shear stress $\tau$ is required for a larger $\Phi$. Smaller $a$ means higher sensitivity of slip velocity to the applied stress, which makes the behavior closer to the traditional strictly threshold-type friction. Throughout this paper, we call $\Phi$ either state or strength, depending on the context.

Although 'stress' and 'strength' are clearly different concepts in material science, the distinction was not well aware of in conventional RFS literature, where the shear stress (or that normalized by the normal stress) was often called the 'strength'. However, the distinction is important in the present paper because a large precursory change of strength under a more or less constant stress is a key ingredient. We follow the conceptual distinction by Nakatani (2001) where strength is an interface property exclusively determined by the physical state of the interface at the moment. As defined in Equation 1, we adopt a notation to represent the state $\Phi$ in the dimension of strength (unit, MPa), not time (unit, sec). This choice is underlain by the notion that the memory of the past is not held by a stopwatch but rather in the form of the current physical state of the interface, most likely the real contact area, which is set as a result of cumulative action of various processes such as aging-induced healing and slip-induced weakening.

The second equation is called the evolution law, which phenomenologically describes changes of the state $\Phi$ for various reasons. Two popular models of the evolution law have been proposed. One is called the aging law or the Dieterich law (e.g., Beeler et al. 1994) that 
explains time-dependent healing very well as observed but has trouble in reproducing an exponential change of friction over a fixed slip distance as repeatedly observed in experiments (e.g., Ruina 1983; Nakatani 2001). Another is called the slip law or the Ruina law (e.g., Beeler et al. 1994), which is good at reproducing the exponential slip-dependent change of friction but fails to reproduce the truly time-dependent healing, contradicting experiments (e.g., Beeler et al. 1994; Nakatani and Mochizuki 1996).

Recently, a revised version of RSF (called the 'Nagata' law hereafter) has been proposed, where the abovementioned shortcomings of the existing RSFs have been largely eliminated (Nagata et al. 2012). It is written as

$$
\begin{aligned}
\frac{d \Phi}{d t}= & \frac{b \sigma}{L} V_{*} \exp \left[-\frac{\Phi-\Phi_{*}}{b \sigma}\right]-\frac{b \sigma}{L} V-c \frac{d \tau}{d t} \\
\text { (the Nagata law) } &
\end{aligned}
$$

where $L$ is a characteristic length scale and $\Phi_{*}$ is a reference state. It is based on the aging law, sharing the first two terms. The first term represents the logarithmic time-dependent healing and $\Phi$ increases by $b$ per $e$-fold increase in the time of stationary contact. The second term represents the linear slip-weakening at a constant rate $b / L$ per unit slip. The third term is the newly found shear stress effect, which is added to the traditional aging law with an empirical constant $c$. On the basis of new rigorous methods of laboratory data analysis, $a$ and $b$ were constrained to be about 0.05 , about five times larger than previously believed and $c$ was constrained to be about 2.0.

Consequences of the Nagata law have been recently shown on the behavior of a single degree of freedom elastic system (Kame et al. 2012a; 2012b) and on the behavior of nucleation on faults embedded in an elastic continuum (Kame et al. 2013a; 2013b). Note that the Nagata law did reproduce the observed $\Phi\left(=\tau-a \sigma \ln \left(V / V_{*}\right)\right)$ very well both in the hold slide and velocity step tests (Figure 1 ) under the same set of parameter values, which was never realized by the existing RSF versions. In this regard, it may be said that earthquake cycle models with the revised RSF more reliably simulate the strength history.

\section{Methods}

\section{Strength drop as an earthquake precursor predicted in RSF simulations}

Using a simple spring-slider model, Kame et al. (2012a) simulated stick-slip earthquake cycles with the revised RSF and carefully looked at the history of $\Phi$. Figure 2 shows the simulated histories of the slip $\delta$, the normalized $\operatorname{logarithmic}$ slip velocity $\log \left(V / V_{*}\right)$, the shear stress $\tau$ and the strength $\Phi$ ( $\tau$ and $\Phi$ are measured from their reference values) during a recurrence interval of 100 years under a constant loading speed (Figure 2, left column), and their 3-year close-ups (Figure 2, right column) focusing on the preslip period lasting for about 2 years in the later stage of a stick-slip cycle, where the slip velocity accelerates from $10^{-9} \mathrm{~m} / \mathrm{s}\left(=V_{*}\right.$, the loading speed) to $10^{-3} \mathrm{~m} / \mathrm{s}$ (an arbitrary threshold to define the onset of an earthquake, $t=0$ ). In the preslip period, the strength decreases under the nearly constant shear stress, and the velocity accelerates following Equation 1. By the end of this period, the strength drops by $\Delta \Phi^{\text {pre }}=76 \mathrm{MPa}$ and the stress drops by $\Delta \tau^{\text {pre }}=4.9 \mathrm{MPa}$ (Table Two in Kame et al. 2012a). The small $\Delta \tau^{\text {pre }}$ reflects the small preslip $\Delta \delta^{\text {pre }}$, noticing that $\Delta \tau=k \times \Delta \delta$, where $k$ is the stiffness of the spring. They are small because of the very slow velocity in this period. On the other hand, $\Delta \Phi^{\text {pre }}$ is fairly large because it reflects $\Delta \ln V$, not $\Delta V$.

In terms of detectability during the quasi-static preslip period, this logarithmic velocity dependency of the strength is very advantageous because the velocity changes by orders of magnitude but remains in a very low absolute range (Kame et al. 2008; Nagata et al. 2012a). A short-term preslip is sometimes thought to be a key precursor of the impending earthquake and has been extensively examined in earthquake cycle simulations (e.g., Kato and Hirasawa 1999; Kato and Tullis 2003). However, the simulated preslip is usually so small that the resulting crustal deformation is difficult to detect at geodetic stations on the Earth's surface far from the preslipping fault at depth.

As long as $V$ is so small that $\tau$ does not change much, the logarithmic sensitivity of $\Phi$ to $V$ is a direct consequence of the constitutive law of RSF as

$$
a \sigma \Delta \ln V=\Delta(\tau-\Phi),
$$

which is independent of the version of the evolution law. Accordingly, a significant strength drop occurred also in the previous RSF simulations with the aging law or the slip law. Although the strength $\Phi$ was not explicitly plotted in those studies, it equivalently appeared as significant increases in the logarithmic velocity in the preslip period whereas the stress was nearly constant because of insignificant preslip (e.g., Kato and Tullis 2003). For example, in the quasi-dynamic simulation of earthquake cycles on a planar fault embedded in a 3D elastic continuum conducted by Kato (2008), the logarithmic slip velocity significantly increased first at the margin of a large circular asperity and then at the central part, indicating a gradual expansion of the region of decreased strength (see the asperity 'LA1' in Figure Five from (b) to (c) and from (q) to (r) in Kato 2008).

\section{Acoustic monitoring of the frictional strength Laboratory experiments}

In laboratory experiments, the slip velocity $V$, the shear stress $\tau$, and the normal stress $\sigma$ of the slipping surface 

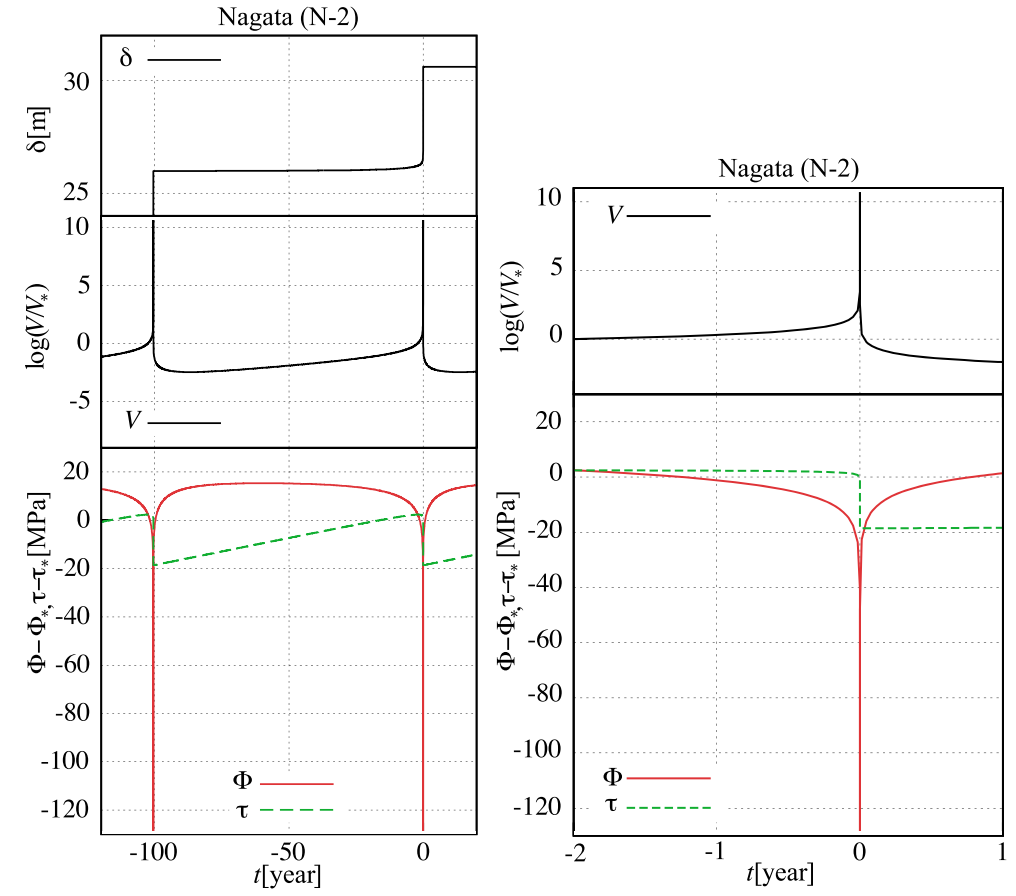

Figure 2 Simulated histories. (Left) Simulated histories of the slider displacement $\delta$ (top), the slider velocity normalized by the load point velocity $V / V_{*}$ (middle), the state $\Phi$ and the shear stress $\tau$ (bottom) for the the Nagata RSF, where $\Phi_{*}$ and $\tau_{*}$ are reference values from steady-state sliding at $V_{*}$. (Right) Simulated histories for 2 years prior to instability at $t=0$. From Figure Five $b$ and Figure Six $b$ in Kame et al. (2012a). Adopted RFS parameters $(a, b, c, L)=(0.051,0.0565,2.0,0.033 \mathrm{~m})$ and simulation parameters $\left(V_{*}, \sigma, k / k_{c}, v\right)=\left(1.4 \times 10^{-9} \mathrm{~m} / \mathrm{s}, 100 \mathrm{MPa}, 0.90,8.8 \times 10^{-5}\right.$ $\mathrm{m} / \mathrm{s} / \mathrm{MPa}$ ), where $\sigma$ is the normal stress, $k / k_{c}$ is the ratio of the spring stiffness to the critical stiffness and $v$ is the coefficient of the radiation damping term.

are observed and the frictional strength $\Phi(=\tau-$ $a \sigma \ln V / V_{*}$ ) at a given moment can be known (Figure 1). Independently, $\Phi$ can be monitored acoustically. Nagata et al. $(2008 ; 2012)$ have conducted rock friction experiments in a double direct shear apparatus, where they simultaneously measured the P-wave transmissivity $|T|$ across the frictional interface using a $1-\mathrm{MHz}$ transducer to monitor the state of contact. $|T|$ showed an excellent agreement with the contact state $\Phi$ (Figure 1). Essentially, the same results have been obtained with S-wave (Nagata 2006) and for an interface separated by a gouge layer (Nagata 2009).

\section{LSM in acoustic methodology}

Acoustic monitoring of the mechanical properties of an imperfectly welded interface was pioneered by Kendall and Tabor (1971) and has been mainly studied in engineering (e.g., Schoenberg 1980). In such acoustic monitoring, the LSM has been employed as a basic mechanical model for wave propagation across an interface (e.g., Schoenberg 1980; Pyrak-Nolte et al. 1990). Here, LSM and its mechanical background are briefly summarized.

An imperfectly welded interface is supported only by asperity contacts. When the thickness of this poorly bonded zone is much smaller than the monitoring wavelength, LSM takes the zone as a zero-thickness interface across which the traction is continuous but the displacement is not. The specific stiffness of the interface is introduced as

$$
t=\kappa \Delta u
$$

where $\Delta u$ is the displacement discontinuity across the interface and is assumed to depend linearly on the traction $t$ with the specific stiffness $\kappa$ (Schoenberg 1980; PyrakNolte et al. 1990). 'Slip' appearing in LSM actually means this displacement discontinuity. The boundary condition for theoretical problems of wave propagation across the interface is completely described by Equation 4 .

Considering elastic deformation of individual asperity contacts, $\kappa$ is represented as

$$
\kappa=N c E r=N \kappa_{0},
$$

where $N$ is the number of contacts per unit area, $c$ is a constant around 2 that depends on the Poisson's ratio, $E$ is the Young modulus of the bulk material, $r$ is the characteristic contact radius, and $\kappa_{0}=c E r$ (unit, $\mathrm{Pa} / \mathrm{m}$ ) is the stiffness due to a single contact (Kendall and Tabor 1971; Yoshioka and Iwase 2006). 
The coefficients of transmission $|T|$ and reflection $|R|$ to a normal incident wave have been theoretically obtained by LSM as

$$
\begin{aligned}
& |T|=\sqrt{\frac{1}{1+\left(\frac{\omega}{\omega_{c}}\right)^{2}}}=\sqrt{\frac{\left(\frac{\omega_{c}}{\omega}\right)^{2}}{1+\left(\frac{\omega_{c}}{\omega}\right)^{2}}}, \\
& |R|=\sqrt{\frac{\left(\frac{\omega}{\omega_{c}}\right)^{2}}{1+\left(\frac{\omega}{\omega_{c}}\right)^{2}}}=\sqrt{\frac{1}{1+\left(\frac{\omega_{c}}{\omega}\right)^{2}}},
\end{aligned}
$$

where

$$
\omega_{c}=2 \kappa / Z
$$

is the characteristic angular frequency, $Z$ is the acoustic impedance of the surrounding medium, $\left(\omega / \omega_{c}\right)$ is the normalized angular frequency. Note that $|T|^{2}+|R|^{2}=1$ because of energy flux conservation in the direction normal to the interface, so $|T|$ and $|R|$ are complementary. In the limits of $\kappa \rightarrow 0$ and $\kappa \rightarrow \infty,|T|$ and $|R|$ give conventional results for a free surface and a perfectly welded interface, respectively. LSM has been applied not only to normal incident $\mathrm{P}$-waves problems but also to problems of seismic $\mathrm{SH}$ and P-SV waves with an arbitrary incident angle, and general solutions for $|T|$ and $|R|$ have been derived (Schoenberg 1980; Pyrak-Nolte et al. 1990).

Figure 3 shows dependency of $|T|$ and $|R|$ on $\left(\omega / \omega_{c}\right)$. In both, a nearly full swing change occurs over a limited frequency range of $\left(\omega / \omega_{c}\right)$ from 0.1 to 10. For effective monitoring, therefore, observation frequency $f$ should be chosen within a two-order bandwidth centered at $f_{c}\left(=\omega_{c} /(2 \pi)\right)$ as

$$
0.1<\frac{f}{f_{c}}\left(=\frac{\omega}{\omega_{c}}\right)<10 .
$$

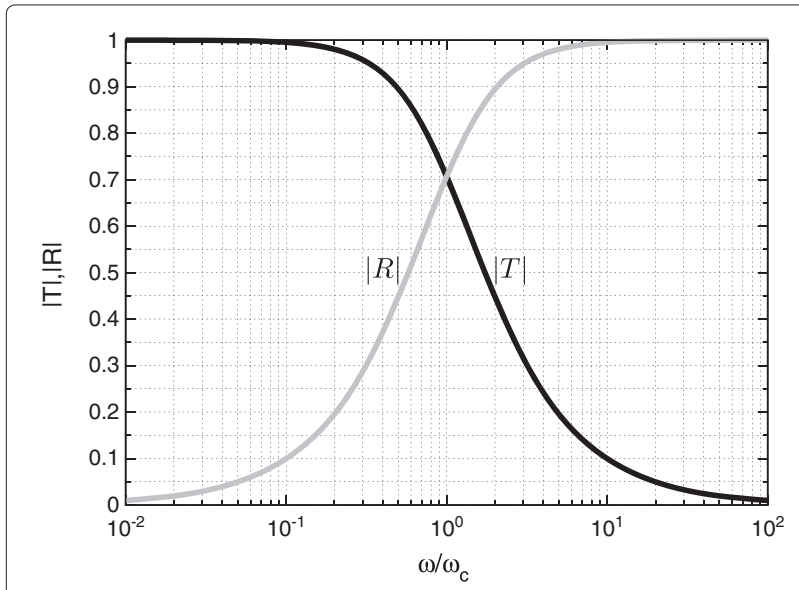

Figure 3 Dependency of the coefficients of transmission $|T|$ and reflection $|R| \cdot|T|$ and $|R|$ depend on the normalized angular frequency $\left(\omega / \omega_{c}\right)$, where $\omega_{c}$ is the characteristic angular frequency.

\section{Relationship between $\kappa$ and S}

LSM relates $\kappa$ to $|T|$ and $|R|$ uniquely. However, $\kappa$ is not necessarily uniquely related with the real contact area $S$ (i.e., $\Phi)$ because $\kappa$ and $S$ depend on $N$ and $r$ differently, as $\kappa=N c E r$ and $S=N \pi r^{2}$ (Kendall and Tabor 1971). Here, we examine how $\kappa$ depends on $S$.

Figure 4 shows four different contact states specified by $\left(N_{i}, r_{i}\right)$, respectively $(i=1,2,3,4)$. If we compare the first two different contact states, $\left(N_{1}, r_{1}\right)$ in Figure $4 \mathrm{a}$ and $\left(N_{2}, r_{2}\right)$ in Figure $4 \mathrm{~b}$, where their contact areas are the same $\left(S_{1}=S_{2}\right.$, i.e., $\left.N_{1} \pi r_{1}^{2}=N_{2} \pi r_{2}^{2}\right)$ but their radii are different by a ratio $I\left(=r_{2} / r_{1}>1\right)$, then $N_{2}$ is smaller than $N_{1}\left(N_{2}=N_{1} / I^{2}\right)$ and the specific stiffness $\kappa_{2}$ is smaller than $\kappa_{1}\left(\kappa_{2}=N_{2} c E r_{2}=\left(N_{1} / I^{2}\right) c E\left(I r_{1}\right)=\kappa_{1} / I\right)$.

The nonuniqueness may be annoying in that $\kappa$ and in turn $|T|$ are not an ideal proxy of $\Phi$ (which is proportional to $S$ ) in acoustic monitoring. Here, we explore two extreme scenarios, $\left(N_{3}, r_{3}\right)$ and $\left(N_{4}, r_{4}\right)$, in increasing the contact area by a factor of $J(>1)$ from the initial state $\left(N_{1}, r_{1}\right)$. If the number of contacts increases by a factor of $J$ while the radius is kept unchanged (Figure 4c), the specific stiffness $\kappa_{3}=N_{3} c E r_{3}=\left(J N_{1}\right) c E r_{1}=$ $J \kappa_{1}$. Consequently, $\kappa \propto S$. In another scenario where the radius increases while the number of contacts is kept unchanged (Figure 4d), the specific stiffness $\kappa_{4}=$ $N_{4} c E r_{4}=N_{1} c E\left(\sqrt{J} r_{1}\right)=\sqrt{J} \kappa_{1}$. In this case, $\kappa \propto \sqrt{S}$. Such different dependencies were verified in laboratory experiments where $S$ was increased according to the two scenarios (Kendall and Tabor 1971).

The above two are end-members and contacts on an actual interface may change in a combined way, which can be constrained by laboratory experiments. This question was addressed by Dieterich and Kilgore (1994; 1996), who showed that as the interface aging proceeded, the contact area increased both by forming new contacts and by merging existing contacts. Since the merged contacts grow in size, while the new contacts are generally small, the typical contact radius remains unchanged. This implies that the increase in the contact area is largely attributed to the increase in the number of contacts.

Furthermore, a theoretical study also supports the constancy of the contact radius in frictional sliding (Yoshioka and Iwase 1996). Yoshioka and Iwase (1996) studied a micromechanical contact model of frictional interfaces, where initial distributions of the contact radius were assumed. The response of the distribution to a velocity change was analyzed both theoretically and numerically. It was shown that some of the existing contacts faded away, being replaced by new asperity contacts, and consequently the radius distribution was unchanged. Their analysis also has shown that the average contact radius $r$ is comparable to the characteristic slip distance $L$ and thus explains why the characteristic displacement $L$ is nearly constant in velocity step tests. They also could explain why the 

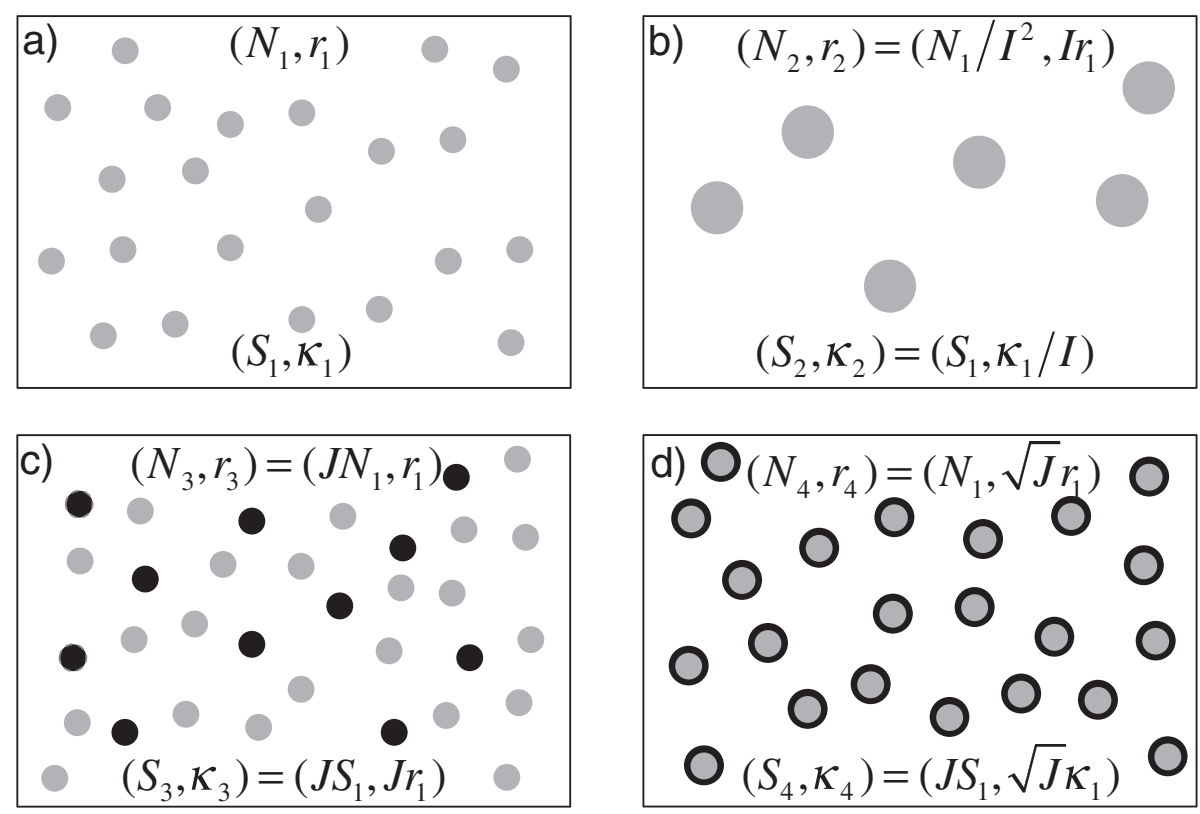

Figure 4 Schematic illustrations of four different distributions of multi-asperity contacts on a frictional interface. (a) A reference state of contact with $\left(N_{1}, r_{1}\right)$, where $N$ and $r$ are the number of asperities per unit area and the asperity radius, which give the contact area $S=N \pi r^{2}$ and the specific stiffness $\kappa=N c E r$ of the interface. (b) Case with $\left(N_{2}, r_{2}\right)=\left(N_{1} / R^{2}, I r_{1}\right)$, (c) case with $\left(N_{3}, r_{3}\right)=\left(J N_{1}, r_{1}\right)$, and (d) case with

$\left(N_{4}, r_{4}\right)=\left(N_{1}, \sqrt{J} r_{1}\right)$. In each panel, also shown is the resultant $\left(S_{i}, \kappa_{i}\right)$. Circles represent the population of asperity contacts and black portions represent the increased parts of the contact area with respect to Figure 4 a. Here, plotting is done with $(I, J)=(2,1.5)$.

characteristic displacement $L$ (proportional to the contact radius) is insensitive to the normal stress $\sigma$ as observed in laboratory.

\section{Results and discussion}

\section{Feasibility of fault strength monitoring in the field} Characteristic frequencies of natural faults

As mentioned in 'LSM in acoustic methodology', significant dependence of $|T|$ and $|R|$ on the contact state is limited around the characteristic frequency $f_{c}$. We start by estimating $f_{c}$ of natural faults by scaling the laboratory results of Nagata et al. $(2008$; 2012), using a scaling relation of $f_{c}$, as derived below.

Suppose that a natural fault has $I$ times greater $r^{\text {fld }}$ and $J$ times greater $\Phi^{\text {fld }}$ than $r^{\text {lab }}$ and $\Phi^{\text {lab }}$ in laboratory experiments. Because $\Phi$ is proportional to the real contact area $S, S^{\mathrm{fld}}\left(=N^{\mathrm{fld}} \pi\left(r^{\mathrm{fld}}\right)^{2}\right)$ is $J$ times greater than $S^{\mathrm{lab}}(=$ $\left.N^{\mathrm{lab}} \pi\left(r^{\mathrm{lab}}\right)^{2}\right)$, which gives $N^{\mathrm{fld}}=N^{\mathrm{lab}} \cdot J / I^{2}$. The specific stiffness is then given by $\kappa^{\mathrm{fld}}=N^{\mathrm{fld}} \cdot c E r^{\mathrm{fld}}=(J / I) \kappa^{\mathrm{lab}}$. Finally, by Equation 8, we obtain

$$
f_{c}^{\mathrm{fld}}=\left(\frac{J}{I}\right) f_{c}^{\mathrm{lab}} .
$$

The level of $|T|$ observed in the experiments by Nagata et al. $(2008 ; 2012)$ was in a range of 0.7 to 0.8 . From Figure 3, such $|T|$ values indicate that their observation frequency of $1 \mathrm{MHz}$ roughly coincided with the $f_{c}^{\text {lab }}$ of their laboratory faults having $\Phi^{\text {lab }} \sim 10 \mathrm{MPa}$. Though $r^{\text {lab }}$ of their sample is not known, we may use their $L^{\text {lab }} \sim 1 \mu \mathrm{m}$ as a proxy as shown by Yoshioka and Iwasa (1996). For the simulation values of $\Phi^{\mathrm{fld}} \sim 10 \Phi^{\mathrm{lab}}$ and $L^{\mathrm{fld}} \sim\left(10^{5}\right) L^{\mathrm{lab}}$ Kame et al. 2012a, $f_{c}^{\text {fld }}$ is estimated to be $\left(10 / 10^{5}\right) f_{c}^{\text {lab }}=$ $100 \mathrm{~Hz}$. For recently reported large $L^{\text {fld }} \sim 1 \mathrm{~m}$ Hori and Miyazaki 2011, Kato and Yoshida 2011 and weak $\Phi^{\text {fld }} \sim$ $10 \mathrm{MPa}$ (Hasegawa et al. 2011) for the $2012 \mathrm{Mw} 9.0$ Tohoku earthquake, $f_{c}^{\text {fld }}$ would be the order of $1 \mathrm{~Hz}$. Fortunately, such frequencies from 1 to $100 \mathrm{~Hz}$ are actually used in field operations.

\section{Sensitivity to the strength change}

By further examining the behavior of Equations 6 and 7, we can derive some useful insights about the sensitivity of $|T|$ and $|R|$ to the interface strength. Since $|T|$ and $|R|$ are complementary, we basically discuss $|T|$ only, though results will be shown for $|R|$ as well. As explained in Section 'LSM in acoustic methodology', strength affects $|T|$ by modifying $\omega_{c}$. In Figure 5, we plot $|T|$ as a function of $\left(\omega_{c} / \omega\right)$. In evaluating the sensitivity to the strength, or $d|T| / d \Phi$, the primary variable is therefore $\omega_{c}$, whereas its effect on $|T|$ is scaled by the observation frequency $\omega$, which should be taken as a given constant upon taking $d|T| / d \Phi$. For shorthand, we denote $\left(\omega_{c} / \omega\right)$ as $\Sigma$. As readily seen from Equation 6, $|T|$ is a unique function of $\Sigma$. 


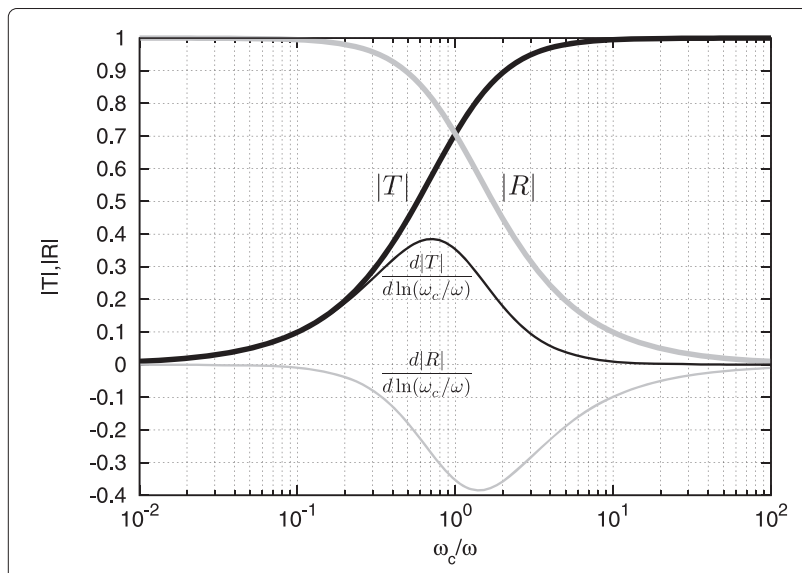

Figure 5 The coefficients of transmission $|T|$ and reflection $|R|$ as a function of $\Sigma \equiv\left(\omega_{c} / \omega\right)$. Their derivatives with respect to $\ln \Sigma$, $d|T| / d\left[\ln \left(\omega_{c} / \omega\right)\right]$ and $d|R| / d\left[\ln \left(\omega_{c} / \omega\right)\right]$, are also shown.

We firstly explore the best observation frequency for monitoring at which the sensitivity to the strength is highest using Figure 5. By noticing that $|T|$ remains approximately 0 when the strength is very low (so that $\Sigma \ll 1$ ) and approximately 1 when the strength is very high (so that $\Sigma \gg 1$ ), it is obvious that the sensitivity depends on the level of the absolute strength. From a practical viewpoint of monitoring a given fault, however, we only need to evaluate the change in $|T|$ occurring upon a strength change from a given initial value $\Phi^{0}$. Since the present paper focuses on fault weakening in the preslip period, we suppose a situation where the strength decreases from an initial value $\Phi^{0}$ to a final value $\Phi^{1}$ by $\Delta \Phi$, though the sense of change does not matter in analysis here. Let us denote the $\omega_{c}$ corresponding to the initial and final states with $\omega_{c}^{0}$ and $\omega_{c}^{1}$, respectively. $\Sigma$ at the initial and final states are $\Sigma^{0}=\omega_{c}^{0} / \omega$ and $\Sigma^{1}=\omega_{c}^{1} / \omega$, respectively. The decrease in $\Sigma, \Delta \Sigma$ is $\left(\omega_{c}^{0}-\omega_{c}^{1}\right) / \omega$, so it depends on $\omega$. As discussed in Section 'Relationship between $\kappa$ and $S$ ', $\omega_{c} \propto \Phi$ seems to be a reasonable assumption for interfaces with natural rough topography like faults (e.g., Scholz 2002). With this assumption, the fractional change in $\Sigma$, i.e., $\Delta \Sigma / \Sigma^{0}$ is equal to the fractional change in strength $\Delta \phi \equiv \Delta \Phi / \Phi^{0}$. This holds at whatever $\omega$. As we are now looking at the sensitivity of a fault with a given strength $\Phi^{0}, \Delta \Phi$ is uniquely related to $\Delta \phi$ and in turn to $\Delta \Sigma / \Sigma^{0}$. Therefore, at whatever $\omega$, the change in $|T|$ and $|R|$, or

$$
\begin{aligned}
& \Delta|T| \equiv|T|\left(\Phi^{1}\right)-|T|\left(\Phi^{0}\right), \\
& \Delta|R| \equiv|R|\left(\Phi^{1}\right)-|R|\left(\Phi^{0}\right),
\end{aligned}
$$

caused by a given $\Delta \Phi$ is known by the changes of $|T|$ and $|R|$ over the same amount of fractional change in $\Sigma$. This means that relative frequency dependency of the monitoring sensitivity $d|T| / d \Phi$ for a fault with a given $\Phi^{0}$ can be evaluated by the slope of $|T|$ plotted against the logarithmic $\Sigma\left(=\log \left(\omega_{c} / \omega\right)\right)$ axis (Figure 5$)$ or equivalently by a function $d|T| / d(\ln \Sigma)\left(=d|T| / d\left[\ln \left(\omega_{c} / \omega\right)\right]\right)$. The same is true for $|R|$, and they are written as

$$
\begin{aligned}
& \frac{d|T|}{d\left[\ln \left(\frac{\omega_{c}}{\omega}\right)\right]}=+\frac{\left(\frac{\omega_{c}}{\omega}\right)}{\left[1+\left(\frac{\omega_{c}}{\omega}\right)^{2}\right]^{3 / 2}}, \\
& \frac{d|R|}{d\left[\ln \left(\frac{\omega_{c}}{\omega}\right)\right]}=-\frac{\left(\frac{\omega_{c}}{\omega}\right)^{2}}{\left[1+\left(\frac{\omega_{c}}{\omega}\right)^{2}\right]^{3 / 2}} .
\end{aligned}
$$

They are plotted in Figure 5 in thin lines. The sensitivity peaks at $\omega=\sqrt{2} \omega_{c}^{0}$ for $|T|$ and $\omega=\omega_{c}^{0} / \sqrt{2}$ for $|R|$. The optimum observation frequencies for a fault with a characteristic frequency of $f_{c}^{0}\left(=\omega_{c}^{0} / 2 \pi\right)$ are as follows:

$$
\begin{aligned}
& f_{|T|}^{0}=(\sqrt{2}) f_{c}^{0}, \\
& f_{|R|}^{0}=(1 / \sqrt{2}) f_{c}^{0} .
\end{aligned}
$$

Inspection of $d|T| / d(\ln \Sigma)$ in Figure 5 reveals that a good sensitivity more than half the peak sensitivity is maintained within a one-order width frequency range around $f_{|T|}^{0}$,

$$
0.5<\frac{f}{f_{c}^{0}}<5 .
$$

The minimum sensitivity in this range is nearly 20 times higher than that for the broader (two-order width) range proposed in Equation 9. Similar performance can be obtained for $|R|$, by setting a frequency range around $f_{|R|}^{0}$,

$$
0.2<\frac{f}{f_{c}^{0}}<2 .
$$

Secondly, we examine the absolute sensitivity or $d|T| / d \Phi$ (unit, $1 / \mathrm{Pa}$ ). We are concerned with the cases where the observation frequency has been chosen appropriately, that is, not too far from $f_{c}^{0}$ corresponding to the strength of the target fault. Under the assumption of $\omega_{c} \propto$ $\Phi, \Sigma$ can be expressed as

$$
\Sigma=\left(\frac{\omega_{c}}{\omega_{c}^{0}}\right) \cdot \frac{1}{\left(\frac{\omega}{\omega_{c}^{0}}\right)}=\phi / \alpha,
$$

where $\phi \equiv \Phi / \Phi^{0}$ is the strength normalized by the initial strength and $\alpha \equiv \omega / \omega_{c}^{0}=f / f_{c}^{0}$ is the observation frequency normalized by the characteristic frequency of the fault at the initial state. Equations 6 and 7 can be written as

$$
\begin{aligned}
& |T|(\phi ; \alpha)=\sqrt{\frac{1}{1+\left(\frac{\alpha}{\phi}\right)^{2}}}=\sqrt{\frac{\left(\frac{\phi}{\alpha}\right)^{2}}{1+\left(\frac{\phi}{\alpha}\right)^{2}}}, \\
& |R|(\phi ; \alpha)=\sqrt{\frac{\left(\frac{\alpha}{\phi}\right)^{2}}{1+\left(\frac{\alpha}{\phi}\right)^{2}}}=\sqrt{\frac{1}{1+\left(\frac{\phi}{\alpha}\right)^{2}}} .
\end{aligned}
$$


In Figure 6, we plot $|T|$ as a function of the normalized strength, for six different observation frequencies around $\omega_{c}^{0}\left(=2 \pi f_{c}^{0}\right)$. The slope of each curve gives $d|T| / d \phi$ or the sensitivity with respect to the normalized strength. The rightmost part of the plot is of foremost concern in appraising the changes in the preslip period, where $\phi$ decreases from the initial value of 1 . For a range of $1>\phi>0.7$, for example, the $|T|$ curves are approximately straight and we obtain an average $d|T| / d \phi$ for this range to be $(+0.02,+0.14,+0.45,+0.45,+0.28,+0.10)$ for the six selected frequencies of $\alpha=\left(10^{-1}, 10^{-1 / 2}, 10^{0}\right.$, $\left.\sqrt{2}, 10^{1 / 2}, 10^{1}\right)$, respectively. At the optimum frequency $(\alpha=\sqrt{2})$ for the fault, for example, $|T|$ decreases by 0.045 for a $10 \%$ reduction in strength. Note that $d|T| / d \phi$ measured at a given $\alpha$, does not depend on the absolute level of strength $\Phi^{0}$, which makes this plot versatile. On the other hand, sensitivity with respect to the dimensional strength $\Phi$, or $d|T| / d \Phi$ (unit, 1/Pa), can be obtained by dividing the $d|T| / d \phi$ by $\Phi^{0}$ because $d \Phi=\Phi^{0} \cdot d \phi$. This means per Pa sensitivity is inversely proportional to the absolute level of strength, even if the observation frequency has been adjusted according to the level of fault strength $\Phi^{0}$, i.e., with $\alpha$ kept constant.

In Figure 7, $|R|$ is plotted against $\phi$. For the six selected frequencies of $\alpha=\left(10^{-1}, 10^{-1 / 2}, 1 / \sqrt{2}, 10^{0}, 10^{1 / 2}, 10^{1}\right)$, $d|R| / d \phi$ averaged for $1>\phi>0.7$ are $(-0.14,-0.37,-0$. $44,-0.37,-0.08,-0.01)$, respectively. Note that the averaged sensitivity at $f_{|R|}^{0}$ is almost the same as that for $|T|$ at $f_{|T|}^{0}$, reflecting the same height of the peaks of Equations 13 and 14. Also, as seen from the symmetry between these two functions plotted in Figure 5, there is no preference between $|T|$ and $|R|$ in terms of sensitivity.

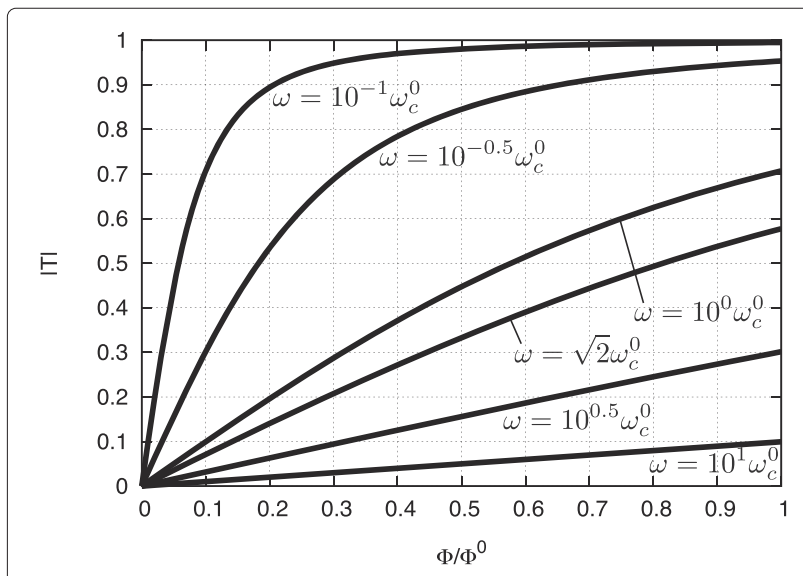

Figure 6 Plots of $|T|$ as a function of the normalized strength $\boldsymbol{\phi} \equiv \boldsymbol{\Phi} / \boldsymbol{\Phi}^{\mathbf{0}}$. For six different reference angular frequencies, where $\Phi^{0}$ is the initial strength.

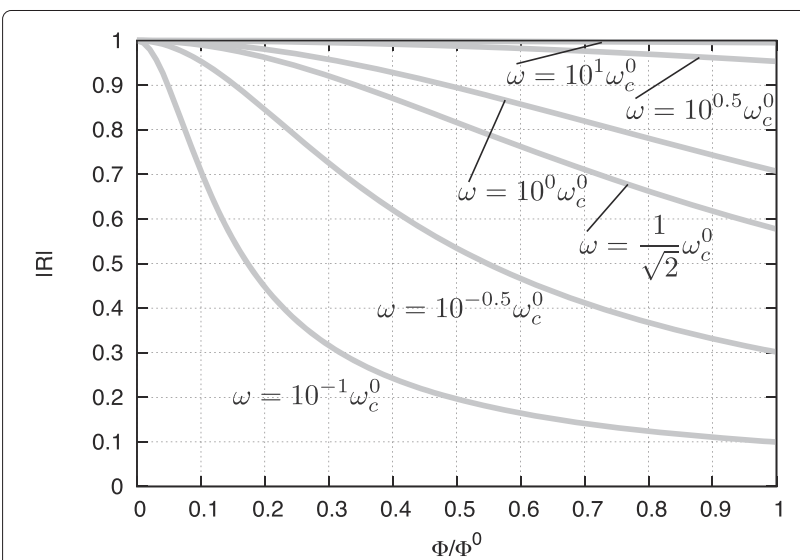

Figure 7 Plots of $|R|$ as a function of the normalized strength $\boldsymbol{\phi} \equiv \boldsymbol{\Phi} / \boldsymbol{\Phi}^{\mathbf{0}}$. For six different reference angular frequencies, where $\Phi^{0}$ is the initial strength.

\section{Magnitude of the changes expected in the preslip period}

The final step is to estimate the magnitude of $\Delta|T|$ to be observed in the preslip period. As mentioned before, $\Delta|T|$ depends on $\Phi^{0}$ as well as $\Delta \Phi$. Parameters in RSF cycle simulations are usually adjusted so as to yield a reasonable stress drop $\Delta \tau$ of an earthquake cycle. Simulations predict $\Delta \Phi$ uniquely according to the model parameters thus constrained, but the absolute level of strength does not affect the simulated behavior at all. So, the simulations give no constraints on $\Phi^{0}$. In addition, the absolute strength level of natural faults cannot be constrained directly by seismic or geodetic observations, and it remains an open question (e.g., Brune et al. 1969; Scholz 2002). So, in the following, we explore cases of both strong and weak faults. We take $\Delta \Phi$ from a simple spring-slider earthquake cycle model Kame et al. 2012a, where a constant stress drop $\Delta \tau=20$ MPa occurred. We assume $\Phi^{0}=30 \mathrm{MPa}$ and $\Phi^{0}=200$ $\mathrm{MPa}$ for weak and strong fault cases, respectively. In their results shown in Figure 2, a $\Delta \Phi \sim 6 \mathrm{MPa}$ occurs for a 1year interval between -1.5 and -0.5 year, and $\Delta \Phi \sim 15$ $\mathrm{MPa}$ for another 1-year interval between -1.1 and -0.1 year. $\Delta \Phi$ becomes further large as time approaches to the earthquake occurrence at $t=0$, where $\Phi$ goes below zero in the weak fault case. This often occurs in RSF earthquake cycle simulations because the employed RSFs do not involve a cutoff velocity suggested for both laboratory and natural faults (e.g., Nakatani and Scholz 2006); the strength drop is theoretically limitless if a cutoff velocity is not implemented. Such an excessive strength drop seems unphysical, so, here, we consider $\Delta \Phi$ only up to $15 \mathrm{MPa}$. At that point ( $t=-0.1$ year), $V$ is still very low, being about 20 times of the plate motion velocity, so that estimating the strength drop with RSF should be still reasonable. Further strength drop would occur beyond 
this $15 \mathrm{MPa}$ mark, but we stick to this conservative estimate of $\Delta \Phi$. For some $15 \mathrm{MPa}$ strength drop expected for -1.1 to -0.1 year, the fractional strength reduction $\Delta \phi$ is 0.5 for the weak fault case and 0.075 for the strong fault case. Using the $d|T| / d \phi$ values obtained in Section 'Sensitivity to the strength change', $\Delta|T|$ for the weak fault is estimated to be $(-0.5) \times(+0.45) \sim-0.23$. This reduction amounts to $40 \%$ of the initial $|T|=0.58$ (Figure 6), which means a $40 \%$ decrease in the amplitude of transmitted wave when observed at the optimum frequency, for example. For the strong fault, $\Delta|T|=(-0.075) \times$ $(+0.45) \sim-0.034$ This reduction amounts to $5.9 \%$ of the initial $|T|=0.58$. The estimates simply scale proportionally with the expected $\Delta \Phi$. As mentioned before, more than half the peak sensitivity is guaranteed if the observation frequency is in the one-order range around the optimum frequency.

The estimate for $\Delta|R|$ goes in much the same way. For the assumed $\Delta \Phi \sim 15 \mathrm{MPa}, \Delta|R| \sim+0.22$ (or $38 \%$ relative increase in the amplitude of reflected wave) for the weak fault and $\Delta|R| \sim+0.033$ (or $5.5 \%$ relative increase in the amplitude of reflected wave) for the strong fault.

Since the Nagata version of rate-state friction was found to have more strength loss for a longer time prior to an earthquake compared with the prediction by the widely used Dieterich law (Kame et al. 2012a), it may be worth mentioning how the feasibility of acoustic monitoring of the precursory strength drop would be affected if the Dieterich law is used instead. By comparing the simulation results obeying the Nagata law and the Dieterich law (Kame et al. 2012a), the strength drop is found to be 5 MPa for the same 1-year interval from -1.1 to -0.1 year, which is one third of the strength drop $15 \mathrm{MPa}$ assumed in the previous estimation using the Nagata law. Accordingly, the corresponding expected changes in $|T|$ and $|R|$ become one third, ranging from a few to a few tens percent of those at the initial state, for which monitoring in the field may be still feasible.

To wrap up, the strength drop during the preslip period likely causes a detectable magnitude of change in the amplitude of elastic waves transmitted through or reflected at the target natural fault. As emphasized in section 'Strength drop predicted in RSF simulations', we think that the estimate of $\Delta \Phi$ is robust or at least conservative. Difficulty is basically proportional to $\Phi^{0}$. If $\Phi^{0}$ is less than several tens of MPa, required resolution on $|T|$ and $|R|$ measurements is fairly tolerant. Accurate measurements with \% level resolution are required for $\Phi^{0}$ of a few hundreds MPa. If $\Phi^{0}$ are nearly GPa or more, detection is too difficult. Also, bear in mind that a higher $\Phi^{0}$ pushes up the suitable frequency range proportionally, so that probing the interface at depth becomes increasingly difficult due to attenuation.

\section{Validation for nature}

Acoustic methods enable us to monitor $\Phi$ remotely. One important point is that $|T|$ and $|R|$ show approximately linear responses to $\Phi$ as shown in Figures 6 and 7 within a range $0.7<\Phi / \Phi^{0}<1$. This means that they are linear to $\ln V$, not $V$. This is very advantageous for preslip where $V$ changes over many orders of magnitude but remains in a very low absolute range. Earthquake cycle simulations suggest that acoustically monitorable changes can be fairly large. The strength drop in the preslip period can be as large as several tens percent of the coseismic strength change over the whole cycle even when the predicted amount of preslip in the same period is only a minuscule fraction of the coseismic slip. In the following, we discuss robustness of our estimates, considering more realistic conditions in the field.

\section{Uncertainties of $r^{\text {fld }}$}

Considerable uncertainties may exist in the value of representative contact scale $r^{\text {fld }}$ (assumed comparable to $L^{\text {fld }}$ ) of natural faults, which strongly affect the estimation of $f_{c}$. Although $L^{\text {fld }}$ up to a meter is now widely accepted among seismologists (e.g., Ide and Takeo 1997; Cocco et al. 2009), they are mostly concerned with the $L^{\text {fld }}$ controlling the dynamic slip-weakening process during an earthquake, which may appear as a result of a mechanism operating only during high-speed slip (e.g., Andrews 2005). Hence, a possibility is left that $L^{\mathrm{fld}}$ relevant to low-speed slip during nucleation is much smaller. However, recently, Kato (2012) has shown that interseismic accumulation of slip deficit for large subduction earthquakes requires a considerable static toughness (fracture energy) of the plate interface. When converted to $L^{\text {fld }}$, this statically high fracture energy implies large $L^{\text {fld }}$ of decimeters to a meter for locked patches of $M 8 \sim 9$ subduction earthquakes, supporting our optimistic estimates.

Even more fundamental criticism to our estimates would be that there is no guarantee that $r^{\text {fld }}$ is approximately equal to $L^{\text {fld }}$. However, we point out that spatially varying amplitude of waves reflected at the plate interface has been already captured by seaboard active reflection surveys conducted over the forearc slope of the Japan Trench (Fujie et. al 2002; Mochizuki et al. 2005). They successfully revealed the intensity distribution of plate boundary $P P$ reflection around 5 to $20 \mathrm{~Hz}$. As we have shown, if one sees $|T|$ or $|R|$ not either approximately 0 or approximately 1 , this means that the employed frequency is not far from $f_{c}$. Unless the observed reflections from the boundary were not made by some special velocity structures, these observed spatial variability of $|R|$ would already mean that $f_{c}$ is in a range practicable in the field. We suggest that this type of survey is worth repeating in search of temporal hanges. 


\section{The nucleation patch on a fault in a 3D continuum}

We have estimated the preslip-induced strength drop in a single degree of freedom spring-slider system (Kame et al. 2012a). Natural faults are embedded in an elastic continuum, and hence the dynamic system has infinite degrees of freedom. Theoretical and laboratory studies have shown that the preslip and associated strength drop on such faults are confined in a creeping patch, which scales with $L^{\text {fld }}$. Acoustic detection is, of course, not possible if the preslip patch is smaller than the resolution of the survey method. Using (indirect) observational evidence, some earlier studies suggest that the nucleation patch is approximately $1 / 10$ of the size of dynamic rupture (e.g., Ellsworth and Beroza 1995; Dodge et al. 1996; Ohnaka 2000). Theoretically, expected size depends on the friction (evolution) law. With the aging law, a fairly large size in an order of $10^{4} \times L^{\text {fld }}$ or more is not difficult to occur (Rubin and Ampuero 2005), consistent with the suggestion by Ohnaka (2000). The Nagata law predicts a smaller but similar size (Kame et al. 2013b), while the slip law predicts a much smaller size of the preslip patch (Ampuero and Rubin 2008).

On the other hand, the magnitude of the strength drop in the preslip patch was not investigated in earlier studies, where detectability was discussed on the slip amount and moment. The simulation of Noda et al. (2013) is an exception in that they plotted the spatial evolution of the strength ( $\Phi$ or in their notation $\sigma \Theta$ ). In their Figure Three $\mathrm{f}$, when the acceleration toward eventual earthquake became clear 3 days before $(0.05 \%$ of their 17 year interval between $M_{w} 6$ simulated earthquakes), the nucleation patch is recognized as a region of about $5 \mathrm{MPa}$ lower strength than the surrounding. This case was simulated with the aging law, but we can expect that the magnitude of strength drop in the accelerating nucleation stage is not strongly dependent on the assumed evolution law or the degree of freedom of the dynamic system. As we pointed out earlier, nucleation necessarily goes through a stage of the by-orders acceleration in the slip velocity under a nearly constant stress drop. Only the spatial extent and the temporal duration of this stage is affected much by the details of the friction law and the degree of freedom of the dynamic system.

We, however, note one important difference between a single degree of freedom system and a fault in an elastic continuum. In the latter, more realistic system in a 3D elastic continuum, the region of strength drop or creep invades the locked patch (asperity) from the perimeter (e.g., Kato 2008; Noda et al. 2013). This stage is not confined in the compact zone of the final-stage nucleation but rather broad, and as Noda et al. (2013) noted, it is not a monotonic acceleration toward catastrophe but can decelerate and reaccelerate along the way. In other words, transient aseismic events can occur for multiple times during this middle stage of an earthquake cycle. From Figure Three of Noda et al. (2013), the magnitude of strength drop in these transients is a few megapascal, compared with the strength in the virtually locked stage early in the seismic cycle. This means that acoustic monitoring would have difficulty in distinguishing these aseismic transients which may decelerate without directly leading to an earthquake from the final-stage compact nucleation that monotonically accelerates toward an earthquake. We think that this likelihood of false alarms is the most important point neglected by our arguments based on a single degree of freedom system.

\section{Seismic velocity versus amplitude}

In this paper, the acoustic transmission/reflection amplitude is proposed as an alternative to the conventional travel time in the monitoring of precursory changes. The possibility of precursory changes in seismic wave speed has been suggested by some earlier studies. Field (e.g., Niu et al. 2008) and laboratory studies (e.g., Kaproth and Marone 2013) show a reduction in the seismic speed preceding an earthquake or laboratory stick slip events. In monitoring the changes of the seismic travel time, however, the difficulty is how to resolve the path effects traveling inside/outside a target fault zone. Niu et al. (2008) conducted active source monitoring at the Parkfield SAFOD drill site where the source and receiver were both installed very close to the San Andreas fault zone at 1 $\mathrm{km}$ depth, reducing the path effect outside the fault zone.

Regarding the seismic amplitude, use of reflected waves may have some practical advantages over transmitted waves in the field though there is no preference between $|T|$ and $|R|$ in terms of sensitivity (Section 'Sensitivity to the strength change'). The amplitude of acoustic waves are affected by both the fault interface and the path in the bulk, but the easily identifiable reflection phase helps single out the interface effect. Also, in dense reflection surveys, spatially coherent patterns, as expected for the nucleation by a preslip patch (e.g., Kato 2008; Noda et al. 2013), may be identified more easily even if the amplitude change at each point is difficult to conclude.

\section{Postseismic fault healing}

As well as the strength drop, significant strength healing is predicted on RSF faults, which also can be monitored acoustically. It has to be noted, however, that $\Phi$ goes back to a certain level in a very short period because the healing is logarithmic with time. Figure 2, for example, shows that postseismic healing of $\Phi$ virtually completes within half a year, whereas $\Phi$ drops gradually over a 2-year preslip period. In order to detect the rather rapid strength healing of the slipped interface, field observations have to be deployed very rapidly. 


\section{Fault gouge}

The present paper explored how changes in the contact area $S$ will lead to changes in the stiffness $\kappa$ for a frictional interface composed of nominally bare surfaces (Section 'Relationship between $\kappa$ and $S$ '). However, many fault zones contain granular fault gouge. Boring cores of natural faults show ultra cataclasite layers of centimeters to meters in thickness (e.g., Chester et al. 2005; 2006). This may bring other possible micromechanics responsible for changes in $\kappa$ : changes of the networks of force chains in a granular layer. This has been studied both experimentally and theoretically, and the amplitude of the transmitted waves has been shown to significantly decrease at the onset of the precursory slip leading up to failure (e.g., Yoshioka and Sakaguchi 2006). This micromechanics can be analogous to changes in the contact number and the contact area between bare surfaces but not directly dependent on the characteristic contact scale. For a fault zone containing a compliant gouge layer, the net interface stiffness becomes smaller (Schoenberg 1980) leading to a lower $f^{\text {fld }}$, which would augment our optimism.

\section{Conclusions}

We explored the feasibility of detecting the strength drop of natural faults during the preslip period predicted in RSF earthquake cycle simulations, by means of field measurements of the amplitude change of transmitted and reflected waves. Combining the contact mechanics of a frictional interface with the LSM in the acoustic methodology, we obtained quantitative relationships between the frictional strength $\Phi$ and the acoustic transmissivity $|T|$ and reflectivity $|R|$. We then estimated the sensitive frequency range to monitor the strength change of natural faults to be about 1 to $100 \mathrm{~Hz}$, suggesting that such monitoring is practicable. Expected changes during the preslip period for years to months prior to an earthquake, when the slip velocity is still minuscule on a linear scale, are about several to several tens percent depending on the absolute level of the fault's strength. Our analysis suggests that precursory strength drop may be large enough to be detected by acoustic survey methods practicable in the field, unless the fault is stronger than several hundred megapascals. We propose that reflectivity/transmissivity monitoring of faults for physical earthquake precursor (reduction in the real contact area) is a quantitatively realistic goal.

\section{Competing interests}

The authors declare that they have no competing interests.

\section{Authors' contributions}

NK carried out the mathematical analysis and drafted the manuscript. KN participated in the design of the study and carried out the mathematical analysis. MN participated in the design of the study and drafted the manuscript. TK carried out the mathematical analysis and drafted the manuscript. All authors read and approved the final manuscript.

\section{Acknowledgements}

Comments by two anonymous reviewers are greatly appreciated. NK was supported by MEXT KAKENHI 21107007. This study was also supported by MEXT, under its Observation and Research Program for Prediction of Earthquakes and Volcanic Eruptions.

\section{Author details}

${ }^{1}$ Earthquake Research Institute, the University of Tokyo, 1-1-1 Yayoi, Bunkyo-ku, Tokyo 113-0032, Japan. ${ }^{2}$ Now at Ministry of Education, Culture, Sports, Science and Technology, 3-2-2 Kasumigaseki, Chiyoda-ku, Tokyo 100-8959, Japan.

Received: 3 January 2014 Accepted: 16 May 2014

Published: 28 May 2014

\section{References}

Ampuero J-P, Rubin AM (2008) Earthquake nucleation on rate and state faults aging and slip laws. J Geophys Res. doi:10.1029/2007JB005082

Andrews DJ (2005) Rupture dynamics with energy loss outside the slip zone. J Geophys Res. doi:10.1029/2004JB003191

Beeler NM, Tullis TE, Weeks JD (1994) The roles of time and displacement in the evolution effect in rock friction. Geophys Res Lett 21: 1987-1990

Brune JN, Henyey TL, Roy RF (1969) Heat flow, stress, and rate of slip along the San Andreas fault, California. J Geophys Res 74: 3821-3827

Chester JS, Chester FM, Kronenberg AK (2005) Fracture surface energy of the Punchbowl fault, San Andreas system. Nature. doi:10.1038/nature03942

Cocco M, Tinti E, Marone C, Piatanesi A (2009) Scaling of slip weakening distance with final slip during dynamic earthquake rupture. In: Fukuyama $E$ (ed) Fault-Zone properties and earthquake rupture dynamics. Elsevier Academic Press, Burlington

Dieterich JH (1979) Modeling of rock friction 1. Experimental results and constitutive equations. J Geophys Res 84: 2161-2168

Dieterich, J H, Kilgore BD (1994) Direct observation of frictional contacts: new insights for state-dependent properties. Pure Appl Geophys 143: 283-302

Dieterich JH, Kilgore BD (1996) Imaging surface contacts: power law contact distributions and contact stresses in quartz, calcite, glass and acrylic plastic. Tectonophysics 256: 219-239

Dodge DA, Beroza GC, Ellsworth WL (1996) Detailed observations of California foreshock sequences: implications for the earthquake initiation process. J Geophys Res 101: 22371-22392

Ellsworth WL, Beroza GC (1995) Seismic evidence for an earthquake nucleation phase. Science 268: 851-855

Fujie G, Kasahara J, Hino R, Sato T, Shinohara M, Suehiro K (2002) A significant relation between seismic activities and reflection intensities in the Japan Trench region. Geophys Res Lett. doi:10.1029/2001GL013764

Hasegawa A, Yoshida K, Okada T (2011) Nearly complete stress drop in the 2011 Mw 9.0 off the Pacific coast of Tohoku Earthquake. Earth Planets Space 63: 703-707

Heslot F, Baumberger T, Perrin B, Caroli B, Caroli C (1994) Creep, stick-slip, and dry-friction dynamics: experiments and a heuristic model. Phys Rev E 49: 4973-4988

Hori T, Miyazaki S (2011) A possible mechanism of M9 earthquake generation cycles in the area of repeating M7-8 earthquakes surrounded by aseismic sliding. Earth Planets Space 63: 773-777

Ide S, Takeo M (1997) Determination of constitutive relations of fault slip based on seismic wave analysis. J Geophys Res 102(B12): 27379-27391

Kame, N, Fujita S, Nakatani M, Kusakabe T (2012a) Earthquake cycle simulation with a revised rate- and state-dependent friction law. Tectonophysics. doi:10.1016/j.tecto.2012.11.029

Kame N, Fujita S, Nakatani M, Kusakabe T (2012b) Effects of a revised rate- and state-dependent friction law on aftershock triggering model. Tectonophysics. doi:10.1016/j.tecto.2012.11.028

Kame, N, Fujita S, Nakatani M, Kusakabe T (2013a) Effects of a newly incorporated stress-weakening term in a revised RSF on earthquake nucleation In: Proceedings of 13th International Conference on Fracture, S20-004, Beijing

Kame N, Fujita S, Nakatani M, Kusakabe T (2013b) Earthquake nucleation on faults with a revised rate- and state-dependent friction law. Pure Appl Geophys. doi:10.1007/s00024-013-0744-0

Kaproth BM, Marone C (2013) Slow earthquakes, preseismic velocity changes, and the origin of slow frictional stick-slip. Science. doi:10.1126/science.1239577 
Kato N (2008) Numerical simulation of recurrence of asperity rupture in the Sanriku region, northeastern Japan. J Geophys Res. doi:10.1029/2007JB005515

Kato, N (2012) Fracture energies at the rupture nucleation points of large interplate earthquakes. Earth Planet Sci Lett. doi:10.1016/j.epsl.2012.08.015

Kato N, Hirasawa T (1999) A model for possible crustal deformation prior to a coming large interplate earthquake in the Tokai district, central Japan. Bull Seis Soc Am 89: 1401-1417

Kato N, Tullis TE (2003) Numerical simulation of seismic cycles with a composite rate- and state-dependent friction law. Bull Seis Soc Am 93: 841-853

Kato N, Yoshida S (2011) A shallow strong patch model for the 2011 great Tohoku-oki earthquake: a numerical simulation. Geophys Res Lett. doi:10.1029/2011GL048565

Kendall K, Tabor D (1971) An ultrasonic study of the area of contact between stationary and sliding surfaces. Proc R Soc London Series A 323: 321-340

Ma KF, Tanaka H, Song SR, Wang CY, Hung JH, Tsai YB, Mori J, Song YF, Yeh EC, Soh W, Sone H, Kuo LW, Wu HY (2006) Slip zone and energetics of a large earthquake from the Taiwan Chelungpu-fault Drilling Project. Nature. doi:10.1038/nature05253

Mochizuki K, Nakamura M, Kasahara J, Hino R, Nishino M, Kuwano A, Nakamura Y, Yamada T, Shinohara M, Sato T, Moghadham PP, and Kanazawa T (2005) Intense PP reflection beneath the aseismic forearc slope of the Japan Trench subduction zone and its implication of aseismic slip subduction. J Geophys Res. doi:10.1029/2003JB002892

Nagata K (2006) Monitoring frictional strength with acoustic wave transmission. Master Thesis (in Japanese with English abstract). University of Tokyo

Nagata, K (2009) Experimental study of friction behaviors using acoustic in-situ monitoring of frictional interface. Dr Sc Thesis. University of Tokyo

Nagata, K, Nakatani M, Yoshida S (2008) Monitoring frictional strength with acoustic wave transmission. Geophys Res Lett. doi:10.1029/2007GL033146

Nagata K, Nakatani M, Yoshida S (2012) A revised rate- and state-dependent friction law obtained by constraining constitutive and evolution laws separately with laboratory data. J Geophys Res. doi:10.1029/2011 JB008818

Nakatani, M (2001) Conceptual and physical clarification of rate and state friction: frictional sliding as a thermally activated rheology. J Geophys Res 106: 13347-1338

Nakatani M, Mochizuki H (1996) Effects of shear stress applied to surfaces in stationary contact on rock friction. Geophys Res Lett 23: 869-872

Nakatani M, Scholz CH (2006) Intrinsic and apparent short-time limits for fault healing: theory, observations, and implications for velocity-dependent friction. J Geophys Res. doi:10.1029/2005JB004096

Niu F, Silver PG, Daley TM, Cheng X, Majer EL (2008) Preseismic velocity changes observed from active source monitoring at the Parkfield SAFOD drill site. Nature. doi:10.1038/nature07111

Noda H, Nakatani M, Hori T (2013) Large nucleation before large earthquakes is sometimes skipped due to cascade-up - implications from a rate- and state- simulation of faults with hierarchical asperities. J Geophys Res. doi:10.1002/jgrb.50211

Ohnaka M (2000) A physical scaling relation between the size of an earthquake and its nucleation zone size. Pure Appl Geophys 157: 2259-2282

Pyrak-Nolte LJ, Myer LR, Cook NG (1990) Transmission of seismic waves across single natural fractures. J Geophys Res 95: 8617-8638. doi:10.1029/JB095iB06p08617

Rubin AM, Ampuero J-P (2005) Earthquake nucleation on (aging) rate and state faults. J Geophys Res. doi:10.1029/2005JB003686

Ruina A (1983) Slip instability and state variable friction laws. J Geophys Res 88 : 10359-10370

Schoenberg M (1980) Elastic wave behavior across linear slip interfaces. J Acoust Soc Am 68: 1516-1521

Scholz CH (2002) The mechanics of earthquakes and faulting. 2nd ed. Cambridge University press, Cambridge

Yoshioka N, Iwase K (1996) The characteristic displacement in rate and state-dependent friction from a micromechanical point of view. Pageoph 147: $433-453$
Yoshioka, N, Iwase K (2006) A laboratory experiment to monitor the contact state of a fault by transmission waves. Tectonophysics 413: 221-238

Yoshioka N, Sakaguchi H (2006) An experimental trial to detect nucleation processes by transmission waves across a simulated fault with a gouge layer. In: Ip WH, Chen YT (eds) Advances in Geoscience, vol 1 Solid Earth. World Scientific, pp 105-116

doi:10.1186/1880-5981-66-41

Cite this article as: Kame et al:: Feasibility of acoustic monitoring of strength drop precursory to earthquake occurrence. Earth, Planets and space 2014 66:41.

\section{Submit your manuscript to a SpringerOpen ${ }^{\circ}$ journal and benefit from:}

- Convenient online submission

- Rigorous peer review

- Immediate publication on acceptance

- Open access: articles freely available online

- High visibility within the field

- Retaining the copyright to your article

Submit your next manuscript at springeropen.com 